Kairos. Journal of Philosophy \& Science 18, 2017

Center for the Philosophy of Sciences of Lisbon University

\title{
Enactivism, Radical Enactivism and Predictive Processing: What is Radical in Cognitive Science?
}

\author{
Klaus Gärtner \\ Center for Philosophy of Sciences of \\ the University of Lisbon (CFCUL) \\ kgartner@fc.ul.pt \\ Robert W. Clowes \\ Nova Institute of Philosophy (IFILNOVA)/Faculty of Social \\ Sciences and Humanities of the Universidade Nova de Lisboa (FCSH) \\ robert.clowes@gmail.com
}

\begin{abstract}
According to Enactivism, cognition should be understood in terms of a dynamic interaction between an acting organism and its environment. Further, this view holds that organisms do not passively receive information from this environment, they rather selectively create this environment by engaging in interaction with the world. Radical Enactivism adds that basic cognition does so without entertaining representations and hence that representations are not an essential constituent of cognition. Some proponents think that getting rid of representations amounts to a revolutionary alternative to standard views about cognition. To emphasize the impact, they claim that this 'radicalization' should be applied to all enactivist friendly views, including, another current and potentially revolutionary approach to cognition: predictive processing. In this paper, we will show that this is not the case. After introducing the problem (section 2), we will argue (section 3 ) that 'radicalizing' predictive processing does not add any value to this approach. After this (section 4), we will analyze whether or not radical Enactivism can count as a revolution within cognitive science at all and conclude that it cannot. Finally, in section 5 we will claim that cognitive science is better off when embracing heterogeneity.
\end{abstract}

Keywords Enactivism, Radical enactivism, Predictive processing, Mental representations, Mental content, Conceptual revolution.

DOI $10.1515 / k j p s-2017-0003$ 


\section{Introduction}

Recent years have seen much discussion of Radical Enactive Cognition ${ }^{1}$ (REC) (Hutto, 2011, 2015; Hutto, Kirchhoff \& Abrahamson, 2015; Hutto\&Myin 2013). Some of what has been said, demonstrates the conviction that REC is revolutionary and will change cognitive science forever. Such proclamations of radicalism should, however, not be taken at face value, and need to be properly weighed. Rather, one can legitimately ask if REC is really the recipe for a radical or indeed revolutionary cognitive science in the way its main proponents claim.

In Section 2 of the paper we discuss the nature of the claims to radicality of the REC framework. We find these claims rest on the idea that what Hutto \& Myin call 'basic minds' should not be considered content involving minds. The argument for this is substantially based upon the supposed failure of philosophical cognitive science to give an adequate theory of content for such minds. Following this, it is conjectured that cognitive science could be fundamentally transformed if it would embrace a purified and radicalized enactive cognitive science eschewing representation talk for at least basic minds. We find these claims are based upon the proposal and adoption of a putatively unifying methodological approach in cognitive science.

In Section 3, we turn to an alternative theoretical development in cognitive science that might count as radical and even revolutionary, namely Predictive Processing models of Cognition (PPC). The predictive processing framework offers a new mechanistic understanding of the brain and cognition in terms of a hierarchical predictive processing architecture (Clark, 2015b; Friston, 2009; Hohwy, 2013). PPC may be thought to share some of the assumptions and orientation of Enactivism, especially when the emphasis is placed upon it as a dynamic and action based model of the mind (e.g., in Clark, 2015b). The discussion of predictive processing serves a dual purpose in our paper. First, it offers an alternative example for comparison of what it might mean for a programme to be radical in cognitive science. In this case the purported radically

1 The name radical embodied/enactive cognition was originally introduced by Andy Clark (Clark, 1997). 
inheres in the reinterpretation of the mind and many of its faculties in terms of new mechanisms for its realization. Second, in Section 3.2, because Hutto has offered a proposed 'RECTification' of predictive processing in a recent paper (Hutto, 2015) it allows us to ask what, if anything, REC adds to the explanatory value of at least one program in cognitive science that it purports to radicalize. We thus use the attempted RECtification of predictive processing to analyze and evaluate the general strategy of REC. We ask what value there is in attempting to purge the explanatory vocabulary and supposedly conceptually clarify the predictive processing framework and whether this, and related attempts, can themselves add up to a radical program. We conclude that although REC proposes a conceptually terse version of predictive processing it seems highly questionable whether it offers an explanatorily superior version to other alternatives.

Section 4 takes a step back and asks what might be revolutionary cognitive science. Following Thagard (Thagard, 1992), and Hutto's own comments on this, we contend that there are two ways in which a scientific program can count as conceptually revolutionary. ${ }^{2}$ The first kind of revolution is a mechanistic one. In this case, concept change is due to mainly empirical findings based upon construal of cognition in terms of novel underlying mechanisms. The second revolutionary way is methodological. Here, the reason for the revolution in concepts is due to a change from one methodological approach to another. We find that REC appears to be revolutionary in neither of the two ways considered. First, as REC is not actually concerned with underlying mechanisms, it cannot count as a conceptual revolution in the former sense. Second, as REC is nothing more than one of many ways to spell out Enactivism, it does not constitute a conceptual revolution in the latter sense. On these grounds we conclude that REC, despite much rhetoric to the contrary, should not be considered as a revolutionary programme in cognitive science.

Finally, in the concluding section, we will review why claims of revolutionary status for methodological programs in general is probably overblown. Cognitive science is quite heterogeneous and explanatory

2 We mainly consider - following Hutto's lead - Thagard's work (Thagard, 1992) on conceptual revolutions (Hutto, 2015). 
restrictions that may work in one sub-field may not necessarily generalize to others. It is better to acknowledge this fact than look to restrict conceptual experimentation in cognitive science with excessive claims for conceptual uniformity and purity.

\section{Radical Claims and RECtification}

It is important to see that radical enactive cognitive science is a conceptual descendent of the enactivist program in cognitive science (Varela, Thompson \& Rosch, 1991). Undoubtedly the enactivist focus on minds as action producers rather than passive knowers has had widespread (Clark, 1997; Di Paolo \& Thompson, 2014; O’Regan \& Noë, 2001), although far from univocal influence throughout cognitive science. Radical Enactive Cognitive science proposes itself as a way "[...] to cleanse, purify, strengthen and unify [...]" (Hutto, 2015, p. 3) theories and practical research taken its lead from The Embodied Mind. REC takes up a major theme in that book, namely, that the notion of representation has allowed us to misunderstand the nature of intelligence and agents. Moreover only rejecting it altogether, for at least basic minds, will allow cognitive science to progress ${ }^{3}$. REC is imagined as the radicalized and theoretically cleaned up (RECtified) form of Enactivism.

The central dispute between REC and representationalism turns on the question of content. While the former wants to abolish the very notion in the context of basic cognition, the latter tries to save, rehabilitate or show that it continues to do good or irreplaceable work within Cognitive science (Clark \& Toribio, 1994; Clowes \& Mendonça, 2016; Degenaar \& Myin, 2014). However, despite the assertions, it seems that nobody has asked the question of how radical REC really is. In this section, we seek to strip away REC's rhetoric and deconstruct what is claimed.

Many of Hutto and Myin's claims are framed in terms of what they call basic minds. Basic minds are conceived of as essentially interactive and dynamically unfolding systems, which are mainly explicated by Hutto \& Myin in terms of their activities:

3 It is important for what follows that this theme was already explicit in The Embodied Mind (Varela, Thompson \& Rosch, 1991). 
"Catching a swirling leaf, finding one's way through unfamiliar terrain, attending and keeping track of another's gaze, watching the sun rising at the horizon-the vast sea of what humans do and experience is best understood by appealing to dynamically unfolding, situated embodied interactions and engagements with worldly offerings. Where we find such familiar activity we find basic minds." (Hutto\&Myin, 2013, p.ix).

Moreover, basic minds are conceived of as at the core of, and deeply integrated within more complex minds - including human minds. The term also refers to much naturally occurring, and presumably all non-human cognition, although its precise limits are not defined. The reader of Radicalizing Enactivism is however left in little doubt that the core of all cognition and much human cognition is, in this sense, basic.

The crucial idea behind this claim, repeatedly emphasized by Hutto and Myin, is that basic minds are not to be characterized in terms of content ${ }^{4}$. Content is something that makes sense $\mathrm{e}^{5}$, so they claim, only when directing at a restricted class of complex minds. The interpretation and analysis of basic minds through the prism of representation and in terms of content they label 'intellectualism', and they are strictly against it.

The main argument employs the conviction that the representationalist cannot properly ground mental content naturalistically. Hutto and Myin's goal is to undermine the core notion of representation as currently used in much cognitive science. Their main argument is that the representationalist cannot properly ground mental content for basic minds naturalistically. By doing away with the central representationalist concept of content, they think they can, for basic minds and beyond, introduce a revolutionary new research program into Cognitive Science. Claims like the following show their attitude:

$4 \quad$ See especially Radicalizing Enactivism: Basic minds without content. (Hutto \& Myin, 2013).

5 As already noted, at least some human mental states do in fact need to be characterized in terms of content. 
"For those working in the sciences of the mind, these are interesting times. Revolution is, yet again, in the air. This time it has come in the wake of avant-garde Enactive or Embodied approaches to cognition that bid us to reform our thinking about the basic nature of mind. [...] The most radical versions of these approaches are marked by their uncompromising and thoroughgoing rejection of intellectualism about the basic nature of mind, abandoning the idea that all mentality involves or implies content. Call this-the view we defend-Radically Enactive (or Embodied) Cognition-REC for short." (Hutto \& Myin, 2013, p. 1).

They claim that the conceptual clarification achieved by really abandoning representationalism will have radical, even revolutionary impact in cognitive science, thus: "In seeking to clarify the true character of cognition REC aims to promote truly revolutionary ways of thinking about mind and cognition." (Hutto, 2015, p. 13).

Surely, we can all agree that the rhetoric used by RECers is revolutionary, to decide whether or not their program is, however, is up for debate.

Now, without assuming that REC is or isn't radical or revolutionary, what does this approach really tell us? As far as we can see, basically two things. Firstly, it seems quite clear that proponents of the theory think that intellectualism about basic minds is wrong. Siding with Wittgenstein, RECers believe in the primacy of action.

"REC 's account o basic cognition is thus given in terms of active, informationally driven, world-directed engagements, where a creature's current tendencies for active engagement are shaped by its ontogenetic and phylogenetic history. Basic minds target, but do not contentfully represent, specific objects and states of affairs." (Hutto\&Myin, forthcoming, p. 18). 
This means, contrary to representationalists ${ }^{6}$, RECers assume that basic cognition is constituted by the ways organisms engage or interact with the world in a goal-driven manner ${ }^{7}$. Radicals deny the claim that basic cognition entails semantic properties. They insist that mentality is characterized by the active engagement of the organism with objects in the environment based on teleosemiotics (Hutto, 2011, p. 22) or biosemiotics (Hutto, 2015, p. 7). That is to say, the organism has intentions which are directed towards aspects in the world. Those intentions, however, should not be understood in terms of semantical content with truth conditions, they rather consist in content-less informational sensitivity with the environment (Ur-intentionality) (Hutto, 2015; Hutto \& Satne, 2015).

Secondly, REC claims if there is no semantic content with truth conditions in basic cognition, then it makes no sense to talk about vehicles of this content. Thus:

"In doing away with the idea content is a defining feature of basic cognition, REC dissolves the foundational notion of representational cognitive science: viz. the textbook content/vehicle distinction. Simply put, if basic minds lack content, then they lack vehicles that bear content." (Hutto \& Myin, Forthcoming, p. 5).

In conclusion, the essential two claims REC makes are a) there is no content in basic cognition and, therefore, b) there is no content/vehicle distinction. In their view this constitutes the revolution of Cognitive Science. The revolutionary character of this claim is of course what is at issue.

We believe that the radical (even revolutionary) claims of REC will stand or fall by its abilities to shape concrete research programs in cognitive science. We take this to largely be an argument within the embodied mind research program. A research program already deeply committed to the primacy of action and a form of non-intellectualism about mind. Let us consider the matter more closely.

$6 \quad$ Pitt (2013) discusses representationalism in detail.

7 It is important to note that this idea is not necessarily incompatible with representationalism. RECers insist that they specify the organism's engagement in non-representational terms. 


\section{Predictive Processing and REC}

\subsection{Predictive Processing as Mechanistic Rethinking of the Mind}

One area where a RECtification attempt has been recently proposed is on predictive processing models of cognition (Hutto, 2015). Predictive processing models of cognition (hereafter PPC) posit a new understanding of the brain as ceaselessly involved in the task of predicting the activity of its sensory surfaces (Clark, 2015b; Friston, 2010; Hohwy, 2013). The brain on this model is conceived of as a suite of mutually constraining and hierarchically organized prediction machines all constantly involved in anticipating sensory impingements. PPC thus give a radical interpretation to much of the activity of the cortex, which is seen as producing a cascading set of Bayesian models of worldly encounters. These predictions are continually tested against incoming sensory information. The PPC promises to unify large swathes of cognitive science offering new mechanistic understandings of mind that, e.g., may unify many cognitive systems which previously held to require different explanations (Friston, 2010).

PPC rests on the idea of a generative model. A generative model is a probabilistic model produced by a hierarchical set of prediction systems instantiated in the brain which aim to predict the incoming sensory stream by inferring the causal structure of the distal causes behind that stream ${ }^{8}$. These systems aim to make predictions about the sensory perturbations generated by the agent's interactions with the world at a vast variety of temporal and spatial scales. These multiple models constrain each other by constant mutual interaction. "Sensory" data coming into the system is now not understood as being translated into percepts, pacé traditional cognitivism. Rather energetic impingements on sensory surfaces are patterns to be predicted or 'explained away'. Sensory signals are only transmitted up the processing hierarchy when they have failed to be adequately predicted by higher levels. When this occurs, these "error signals" propagate up to higher level systems of processing which use

8 See Clark, 2015b (Chapter 1: Prediction Machines) for a layman's introduction to these models and their meaning. 
them to refine the suite of generative models either by improving the precision of existing models or by generating new ones.

At the lowest levels, neural models predict micro-domains of perturbation such as whether a line is scanned by the retina at a certain orientation, whereas higher levels predict the activities of systems lower down the hierarchy and, indirectly large scale features of the perceptual field. Crucially, much of the information flow in the brain is to be understood of in terms of higher level systems predicting the activity of those systems which are lower in the hierarchy and closer to sensory activity. In contrast with traditional versions of hierarchical processing in the brain information is only fed forward when it surprisingly disconfirms the predictions and expectations of the brain's more global models.

Perception, according to PPC, is understood in quite different ways on PPC to standard cognitivism. The brain is not conceived of attempting to transduce information from the senses and then fuse it to produce an overall representation. Rather it is conceived of as ceaselessly involved in the prediction of sensory engagements. Each act of perception begins with the current best guess about the state of the world and uses a hierarchical generative model to produce an effective simulation of the causal structure behind the incoming sensory stream. The mind is now understood, at its highest levels, as inferring or projecting an overall virtual model which is continually tested and refined through the current sensory perturbations of the agent, including the agent's active exploration of its environment (Clark, 2015a). All this prediction works to find a causal model of whatever set of causes would most adequately predict the sensory data. As Clark puts it, "We recognize objects and states of affairs, if these approaches are correct, by finding the most likely set of interacting factors (distal causes) whose combination would generate (hence predicts and best accounts for) the incoming sensory data." (Clark, 2015b, p. 21). Perception is conceived of, in words Clark frequently uses in his book, as "richly world revealing".

But the radicalism goes beyond the mechanisms of neural systems as the model reposes much of what tradition psychology posited about the relative independence of the faculties of mind. The same models used in perception to predict sensory stimulations are used "offline" in imagination, dreaming and hallucination. Faculties once thought of as separate 
now come as a common suite. Perception, imagination, action, the emotions, and attention all may now be subsumed into a unified processing system (Clark, 2012a, 2012b; Friston, 2010). Sensory integration and models of attention appear to come for free on these models.

PPC also allow a reinterpretation and new explanation of a host of cognitive phenomena from schizophrenia and delusions (Fletcher \& Frith, 2009; Clowes 2017), to an understanding of binocular rivalry (Hohwy, Roepstorff \& Friston, 2008) to a model of conscious presence (Seth, Suzuki \& Critchley, 2011), to mention just a few. By virtue of this apparent unification of a host of cognitive properties along with many new lines of research, PPC could certainly be argued to be a grand new model of mind.

Even Hutto, concedes the new thinking is radical stating: "PPC represents a dramatic reversal of traditional cognitivist thinking. It regards the core business of cognition to be making pro-active Bayesian predictions about likely sensory perturbations as opposed to constructing internal models of the world that are built upon passively receiving information furnished by the senses." (Hutto, 2015 p. 6). Although, not radical enough.

Despite its new implications, in many respects the PPC in its typical explications is a highly, even ultra-representational, view of the mind (Gładziejewski, 2015). After many years in cognitive science which radically situated and embodied views of cognition claimed that the mind did not require much information integration at all (Brooks, 1995; Brooks \& Stein, 1993) such findings on multiple and multifaceted inner integration may look extremely unexpected. If one was to create a hypothesis about how the brain would work which would vindicate a representational picture-albeit of an unconventional sort-PPC would prima facie seem to be such a picture. The brain appears now to be massively modelling the world at a variety and open-ended set of temporal and spatial scales. These models mutually constrain each other and theoretical terms used by the scientists inventing these ideas make liberal use of terms such as model, representation and content, which also seems to play a central role in their conceptual models ${ }^{9}$.

$9 \quad$ See for instance the frequent use of the term representation in explicating the model in (Friston, 2010). The point here is not that this indicates representation is the only 
And yet standard accounts make much use of the terms model and Bayesian predictions. Indeed, Bayesian prediction is often thought of just a form of model making, just in a different way to which most cognitivists originally thought about how the senses inform those models, i.e. how those models were thought to be formed and deployed. "Rectifying" such a position looks like no easy task and indeed the PPC outlook seems like a highly inhospitable territory to being subsumed by the REC viewpoint. Attempts to RECtify the PPC picture by removing all representational gloss look ambitious indeed and Hutto then indicates that the radical enactivist approach to PPC would be a great achievement precisely because PPC is generally considered representationalist through and through (Gładziejewski, 2015; Hutto, 2015, p. 7).

\subsection{Is RECtifying PPC Radical?}

Hutto's RECtification of predictive processing takes Clark's (2012b, $2015 \mathrm{a}, 2015 \mathrm{~b})$ already rather enactive interpretation of predictive processing as its main target. Hutto's major criticism of Clark ${ }^{10}$ centers on a metaphor Clark uses to explicate the interaction of the functioning of PPC systems. Hutto writes that Clark "asks us to imagine a game in which one participant attempts to describe what a second participant is seeing while the latter moves through a familiar environment - the living room of the first person's house. The catch is that the first player has no direct access to the visual scene and so can only make best guesses about what the second player is likely to see. The second player's role is to speak up and correct those guesses should they go awry and to remain silent otherwise. Hence if player one says "There's a vase of yellow flowers on the table in front of you", the second player will either deny this or remain quiet." (Hutto, 2015, p. 8). Clark's metaphor nicely captures the sense in which higher level systems posit perceptual content which

way such models can be explicated. Indeed, Hutto's work and other recent work points to alternative enactivist possibilities. We are merely pointing out that those formulating the ideas are explicitly using representational notions to explicate and arguably generate their ideas.

10 Hutto also criticizes Hohwy (especially Hohwy, 2014). But as we think that the substance of this part of Hutto's critique is on the right lines, we will focus primarily on the criticisms directed at Clark's views. 
is then confirmed or denied by systems lower down the hierarchy and closer to sensory surfaces.

However, Hutto demands this metaphor must be "cleansed" of its commitment to content. He writes: "[t]he trouble is that anyone hoping to explain what the senses deliver in this way faces a hard choice. Either take talk of rich informational messages and content seriously (and pay for it by answering the Hard Problem of Content directly (by showing how mere covariation adds up to content) or go radical, ditch the idea that information in question is contentful and significantly revise the story." (Hutto, 2015, p. 8). The problem with Hutto's semantic cleansing here is that he doesn't offer us any other intuitive picture to replace what he proposes removing, merely a critique of the naturalistic credentials of representational content.

In any case, we believe Hutto may present these options as unnecessarily stark. Either we give a theory of content or we give up representations. Hutto wants to show that PPC are not only in need of RECtification, i.e., in need of being interpreted in ways that do not refer to contentful inner states. But doing this should presumably give us some clear explanatory advantage. Hutto should show us not only that the REC approach can indeed make sense of and motivate PPC, at least as well as standard approaches, but should in addition show that it offers some explanatory advantages over the representational accounts in which it is usually couched, and within the conceptual framework of which it was first developed.

Hutto argues that:

PPC should not be described in terms of content, because such accounts of content cannot be naturalistically discharged.

The epistemic interpretation of PPC is not necessary. That is, internal Bayesian models should not be interpreted as "getting things write or wrong" because "perceiving isn' $t$ fundamentally a matter of representing the world then there is simply no question of our perceptions getting things right and wrong in basic cases." Moreover, Hutto holds that to claim otherwise is to make the assumption that Cognition must Involve Content (CIC), which is exactly what he is contesting. 
Both arguments go hand in hand. If, as 1) tells us, there is no content, then we need to find a way to make sense of non-epistemic internal Bayesian models, hence 2).

Argument 1) is clearly just Hutto's standard skeptical approach to the "hard problem of content" in Cognitive Science. Hutto points out that there is no accepted theory of content and argues that there is no prospect of one (e.g. Hutto, 2011; Hutto \& Myin, 2013, forthcoming). There is no specificity to this criticism of PPC as it is the very same argument he offers against all contentful models in Cognitive Science (or at least to those that attempt to explain so-called "basic minds").

More specifically, he claims that teleosemantic models do not work as ways of unpacking PPC because "to adopt a teleosemantic account of mental content is to forego the idea that mental contents can possibly feature in mechanistic or causal explanations." (Hutto, 2015, p. 9).

Now, Hutto is possibly right if we consider a standard teleosemantic view where Darwinian adaptation holds ${ }^{11}$. This is because under standard teleosemantics it is the adaptive history of organisms which fix the content producing capacities of any representational mechanisms. Pacé Miłkowski (2016a), this is supposed to set content apart from the actual mechanisms of cognition. Applied to representations - so the standard view goes - teleosemantics can only ever establish the meaning that a content might have played in the adaptive history of the entity. This is taken to preclude giving an account of the content of PPC states precisely because these are always under revision by interaction with the world. But this need not be the case. Teleosemantics need not be interpreted only in terms of classical Darwinian evolution, but could be interpreted in terms of any adaptationist process that could give rise to complex design or organisation.

Consider for instance, the adaptationist robotic systems for constructing word meaning in Luc Steels's language simulations. Steels and his colleagues developed a series of algorithms and interaction protocols for robots - both in simulations and physical robots - which allowed agents

11 Albeit, see the detailed discussion in Miłkowski (2016a). We tend to side with Miłkowski's view that it is the specifics of whatever feature of cognition is under investigation as to whether the content in the particular explanation plays a causal role or not. 
to collectively bootstrap word-meaning through language-games (Steels, 1997; Steels \& Belpaeme, 2005). In these experiments each word meaning was under a continual selection pressure due to the need to have them successfully interpreted by other agents. Hutto's focus on that standard evolutionary story about teleosemantics means that he does not consider the possibility that there are other ways semantic content might be fixed by adaptation, on timescales both more rapid than Darwinian evolution, but also that could be more easily tied to individual psychological mechanisms.

Indeed, the process of refining cognitive models through the ongoing response and integration of error signals in PPC systems could itself be looked upon as an adaptationist process by which an agent's current representational media are progressively made more adequate for tracking and negotiating its active worldly encounters. For an agent whose cognitive apparatus functions according to hierarchical predictive processing, every perceptual encounter may be regarded as an adjudication over the adequacy of its current representational vocabulary to articulate current sensorimotor predictions and encounters. Generative models that fail to predict the senses adequately are altered in ways that are analogous to the way that the genes of animals are culled that fail to reproduce. This putative content is still fixed by the organism's adaptive history, but only partly to be analyzed in terms of more standard Darwinian mechanisms and timescales. One way of underwriting the contentful posits of the system in ways that are causally efficacious are in terms of the adaptive processes by which error is used to correct ongoing predictions.

This is one reason that PPC models of cognition are not just any old representational model, but are ones where representation and representational content are front and centre. Teleosemantics is generally held to be problematic because it does not tell us about the current functional involvement of any mechanism, but merely the functional involvement of the mechanism when it was under selection pressure. But the advantage of cashing out teleosemantic theory in terms of a predictive processing architecture is that each individual sensory engagement of the agent can be understood as part of its adaptive history. Assuming such interaction contributes to the content of any representation means that its 
representational vocabulary is fixed not by a historically distant evolutionary process but the sum of its by sensorimotor engagements, up to and including its most recent encounters. Content on this analysis is not disengaged from mechanism, but defined by the history of an individual organism's engagements. Content in predictive processing systems could be considered to be continually revised in order to articulate the current reality of sensory perturbations. Semantics and mechanism only come apart under teleosemantic theories if we agree that Darwinian natural selection is the only adaptive mechanism in town, and perhaps, as Miłkowski (2016a) argues, not even then. Thus, a teleosemantic account of PPC may not appear to be in anything like as bad a position as Hutto would have us believe.

Argument 2) holds that the epistemic interpretation of PPC is not necessary. Not only, if there is something to the reflections about 1) just entertained, then maybe there is no need for this move. But PPC may well be the mechanistic account of cognition which is most naturally and concisely interpreted in an epistemic way.

The epistemic interpretation of PPC, via the representationalist view of mind, provides reasons to think that semantics and mechanism are closely locked together. For instance, Hohwy interprets every perceptual and action engagement as being a check on the accuracy of current content. As Gładziejewski (2015) observes "prediction error minimization aims to minimize the mismatch between how things are and how the brain/mind "represents" them as being" (Hutto, 2015, p. 10). In comparison to e.g. the computationalist theory of mind this model makes error-checking, and thus the content of representation is central to the whole theory.

As a result, the PPC approach to explaining brain activity seems naturally couched in and understood in content involving terms. On (epistemic) PPC accounts, agents (and their brains) are considered to be making rich predictions about the world which are corrected by their interactions. These predictions are multi-modal and are explicated in terms of their ongoing success or failure in predicting sensorimotor encounters. The idea that inner models are richly predicting the world implies that, at both a basic and more specific and highly elaborated level, gives reality to the semantic richness we ordinarily use in the 
explanatory unpacking of what the brain is doing when agents interact with the world. Given the natural adequacy of epistemic interpretation of PPC, we would need a very good reason to give it up ${ }^{12}$.

Now consider Hutto's solution. In essence his approach states that all that we need to refer to is a hierarchy of statistical models. But this weirdly - for one so committed to an interactionist philosophy of cognitive science - looks like a internalist move. When we take away the semantic posits, we seem to be only left with the maths of brains and their neural networks. This may be fine if our interests are is discretely the understanding the algorithms of brains, but this makes it difficult to make sense of embodied brains dynamicallly interacting with their environment. Something that more naturally falls out of epistemic interpretation. Perversely then the RECtification of PPC seems to move us further away from an enactivist view of mind embedded in, and dynamically producing cognition through its engagements in the world.

The RECTified approach may give us a semantically terser explanatory framework, but it is not clear this has either explanatory or generative advantages ${ }^{13}$. Hutto's rigorously holds to the idea you should not use conceptual vocabulary you cannot cash out, in a way that recalls logical positivism. But science is driven by all sorts of explanatory posits rich and terse. Moreover, it is far from clear that this sanitization is something which really drives cognitive science forward. Rather it may be that a more pluralistic framework is more accommodating, and as we have argued the epistemic interpretation of PPC might be accommodating for new ways of thinking about content even for basic minds.

12 To be clear, we are not suggesting that the brief thoughts here add up to anything approaching a full unpacking of teleosemantics in terms of predictive processing or Bayes theory. However, we hope we have said enough to show that not all avenues are closed for the epistemic interpretation of PPC. Indeed, there are possibly productive interpretations that are yet to be fully explored.

13 As pointed out earlier the formulators of these ideas appear to think in representational terms. It is not clear why RECtifying the "realm of discovery" in the way proposed might be advantageous. Indeed culling conceptual categories could, just as easily, restrict the conceptual resources underlying research. 


\section{Is REC Revolutionary?}

\subsection{Why Think of a Revolution}

At this point we may ask why we need all this discussion of radicalism and revolution? The short answer is that Enactivism and PPC both offer themselves as new and challenging frameworks that might shake-up and dramatically change the way we think of the mind. We need to find some way of determining if these claims are anything more than rhetoric. Hutto and Myin claim that RECtifying explanatory accounts in cognitive science will give us a purified and unified picture. They think that REC is a tool for engineering a conceptual revolution that will change Cognitive Science profoundly (Hutto, 2015; Hutto\&Myin, 2013, forthcoming). How justified are they in this view?

This is what Hutto states:

"Conceptual revolutions are rare, to be sure. Yet following the REC path seems bona fide revolutionary precisely because in doing so it to press for "the replacement of a whole system of concepts and rules by a new system" (Thagard, 1992, p. 6)." (Hutto, 2015, p. 13).

And offers REC's job as analogous to early revolutions in science where fundamental rethinking of categories was required:

"For example, the Copernican revolution also required a fundamental ontological rethink, requiring us to reclassify "the earth as a kind of planet, when previously it had been taken to be sui generis" (Thagard, 1992, p. 36). Likewise, "Darwin did not simply pick away at the creationist conceptual structure: he produced an elaborate alternative edifice that supplanted it as a whole" (Thagard, 1992, p. 36)." (Hutto, 2015, p. 13).

Comparing the new enactive cognitive science to the Copernican and Darwinian revolution sets the bar high. REC is touted not simply as implying a revision about how we think about basic minds, but as a revolution in cognitive science. This means, "[...] in a tree-like hierarchy, it is 
clear that REC presses not merely for conceptual branch jumping within a tree, but switching to a new tree altogether." (Hutto, 2015, p. 13). For Hutto (and Myin) REC has the potential to confirm a scientific revolution that changes mankind's scientific conceptual apparatus. This sets the bar high.

In the context of the PPC at least, this seems to be overstating the case. As shown in the previous section, one can question not only whether Hutto's story is the only one we can tell, but whether it makes the most adequate sense of PPC. But beyond this it is far from clear that the RECtification of PPC offers anything new. At least it is not clear what is new about what is on offer. We think, more generally, the evidence for concluding that REC has the revolutionary potential claimed is rather slim. In this section, we seek to test more generally the claims for revolutionary potential of REC.

As we have seen in the above quotes, it is important for Hutto to highlight what a conceptual revolution consists in. It is worth returning to Thagard's original passage on this question, from which Hutto \& Myin frequently cite:

"I shall count conceptual changes as revolutionary if they involve the replacement of a whole system of concepts and rules by a new system. The two key words here are "replacement" and "system". Merely adding a new set of ideas poses no special problems, and replacement of a single concept or rule should be simple to process." (Thagard, 1992, p. 6 - Highlighted by us).

The crucial point is the highlighted section. At this point, we think that there are different ways we could tackle this issue. For instance, we could discuss how REC replaces the system of representational concepts of CIC and ask whether or not we are better off. We have already tried to argue along these lines in section 3, but we will resist this step here. In our view the real question is whether REC is merely replacing a few sets of concepts or the whole system. To do so, we will focus on the two types of conceptual revolutions, discussed by Thagard, and argue that there are serious doubts that REC is a good candidate to be seen as either. 


\subsection{Will the real revolutionaries please stand up!}

The first kind of conceptual revolution according to Thagard is essentially mechanistic. ${ }^{14}$ This means concept change in a scientific domain is due to new - often empirical - findings which alter the way we think about the underlying mechanism. Take the chemical revolution for example where Stahl's dominant phlogiston theory of combustion is replaced with Lavoisier's principe oxygine (Thagard, 1992). Both theories are conceptual systems which provide an explanation for a variety of phenomena that include processes of combustion and calcination. While the former theory holds that combustion consists in adding phlogiston to e.g. ores to create metals and calcination is subtracting phlogiston, the latter treats both processes the other way around, i.e. combustion means subtracting oxygen and calcination adding it.

The second form of conceptual revolution consists in changes in methodology. Here, alteration in concepts is due to the change from one methodology to another. ${ }^{15}$ This kind of revolution takes place when we consider so called methodological programs, i.e. scientific research that is primarily characterized by a particular methodology. Consider for instance Behaviorism. Historically this approach was designed to break with the introspectionist paradigm of mental facts. It can be characterized as the "[...] elimination of states of consciousness as proper objects of investigation" (Watson, 1913, p. 177). The goal of Behaviorism was to establish "empirical laws" (Thagard, 1992, p. 228). As a consequence, psychology should study a creature's learned habits and the underlying conditioned reflexes. Behaviorist research findings were conceived of as consisting in stating stimulus-response-schemes. This contrasted deeply to the then dominant pre-behavioristic introspective methodology used in psychology designed to study objects of consciousness. Behaviorists first asserted their new methodology in the domain of animal research, where introspectionist research was clearly lacking, but then broadened

14 Thagard describes it as substantial theory replacement which, according to him, happens in the natural sciences. We think that it may well be the case that this type of conceptual revolutions extend to other areas of science.

15 Thagard thinks that rival approaches (not a unifying theory) change due to methodological considerations. In his view this is what happens especially in psychological revolutions. 
their explanatory scope. They insisted that the proper methodological approach of describing empirical Stimulus-Response-Schemes could be expanded to encompass the whole of psychology. These methodological considerations behaviourists argued entailed abandoning concepts such as consciousness, sensations, will or similar notions and replaced them with stimulus, response, conditioning, etc. (Thagard, 1992).

Does REC qualify as revolutionary in either of the two senses given? We think that REC does not fulfill the standards of a conceptual revolution in the former sense. This is because clarifying or radicalizing an already existing view about an underlying mechanism does not change the mechanism. This means, Enactivism introduces new concepts about the mechanisms of cognition, such as interaction, movement and action as opposed to manipulating incoming information. Radicalizing this view may only tell us how we should spell it out, does however not affect these underlying mechanisms.

Now, having a closer look at the ordering relation reveals this important difference. Mechanistic theories fix the conceptual hierarchies within their field. To overthrow those hierarchies means to restructure the fundamental concepts upon which a particular science is based. This has implications for the whole field (Thagard, 1992). Let us look at the chemical revolution as an example. Conceptual systems employing e.g. phlogiston or oxygen are theories about the underlying chemical mechanisms, which structure the hierarchy of concepts. This means, Stahl believed that ores can only become metal by adding phlogiston. However, Lavoisier noticed, in the process of combusting phosphorus and sulfur, that the end product gained weight. Based on this contradiction and other observations, he concluded that it is difficult to account for this mechanism in terms of te former theory. Only replacing essential, fundamental concepts of theories makes a conceptual revolution possible. This is to say - physical reductionism aside - that only the replacement of the theory which describes the underlying mechanism changes the system of concepts and restructures their hierarchy. 
It is far from clear whether or not cognitive science even has a unifying theory in the first place (Miłkowski 2016b) ${ }^{16}$, and we think that it does not $^{17}$. For the sake of argument, however, we will assume for a moment that it does. In this context, this means that the traditional computational view of cognition has to be replaced with a new unifying theory. Now, what would have to change for this to be true? In terms of the traditional computational theory, cognition is conceived of as a passive process of manipulating information (computation). To replace this view, one has to replace the mechanism which describes what cognition is. Consider Enactivism for instance. Varela claims that cognition should be understood as an organism's interaction with its environment (Varela, Thompson $\&$ Rosch, 1991). This has many consequences, e.g. that cognition is essentially active and also that it does not happen only in the head. This is clearly a new way of describing the underlying mechanism of cognition. Therefore, Enactivism may count as an alternative unifying account.

Now, what does Hutto and Myin's RECtification bring to the table? It gets rid of content and hence representations. However, in our view "content" and/or "representation" are not concepts that describe the underlying mechanism. This means they are not on par with concepts like interaction or action. The essential claims to break with the computational view of cognition are already entailed by Enactivism. One indicator is that most representationalists assume that representations, sooner or later, have to be unpacked in naturalistic terms (e.g. Pitt, 2013). This means representations are theoretical constructs, which do not concern the underlying structure of cognition. This constitutes an important, but nevertheless only one, manner of spelling out views of cognition.

One way to show this is to argue that representational descriptions are organized functionally. This means, essentially representationalism collapses into functionalism. In this case, the only differences important

16 Thagard (1992) discusses this issue.

17 The reason is basically that neither computationalism nor enactivism are views about cognition which came about because of (empirical) findings that proved the previous approach wrong - as it is the case with the principe oxygine. Loosely speaking computationalism treats cognition somehow analogous to computation. This was, however, due to methodological considerations. The same applies to enactivism. In our view, both views constitute conceptual revolutions only in the methodological sense. 
to cognition are differences in content detected by their functional role. Kriegel states, in the context of representationalist accounts of the phenomenal, that "[...] [w] hat plays the functional role $D$ [distinctive functional role] is perhaps the mental state that carries $C$ [content], but not $C$ itself. Functional role is not an attribute of representational contents, but of the vehicles that carry these contents." (Kriegel, 2002, p. 60). Of course, RECers want to do away with the content/vehicle distinction and hence representations, however, they do not want to do away with functional roles. Hutto claims that "[a]lthough the senses are sensitive to information in the environment, they can do their action guiding work in a strictly silent manner [...]. There are ways of making sense of the function of the senses in which representational contents play no part [...]." (Hutto, 2015, p. 12). Both views share that what in the end does the job are the functional aspects of cognition. The difference is that the representationalist describes this in terms of content/vehicles, and RECers do not. Consequently, neither representationalism nor the radicalizing elements of REC should be considered to entail concepts of the underlying mechanism, rather they are ways of articulating this underlying mechanism. Therefore, if REC is or is not revolutionary depends on EC (Enactivism) and not R.

In this context, let us apply these considerations to what was said about PPC in the last section. According to Hutto, REC tries to clarify, indeed to "purify" 18 this and other enactivist views. If what was said in the last paragraph is right, radical change, however, is grounded either in PPC or Enactivism and not in radicalizing these theories. Whether a representationalist unpacking or RECtification is applied does not affect the underlying mechanism. Prima facie it does not matter how the underlying theory is spelled out. RECers, however, think of CIC quite literally. In their interpretation this view is committed to hold that cognition is essentially representational. Of course, there are those who believe that (e.g. Fodor, 1981). However, from what was said so far one only has to assume that

18 In Hutto's view this is the whole purpose of REC. He states: "[REC's] analyses and arguments are designed to cleanse, purify, strengthen and unify a whole set of existing anti-representational offerings. REC's aim is to radicalize existing versions of enactivism and related explanatory accounts through a process of philosophical clarification." (Hutto, 2015, p. 3). 
mental representations are nothing more than an analytic tool in trying to understand complex issues. In terms of this approach, we think that there are adequate contexts to use representation talk when referring to basic minds (Clowes \& Mendonça, 2016).

This leaves only the second option. But before we set up the argument let us recall what was said about REC so far. Until now we have already seen that by itself REC is no particular explanatory framework. It is rather a way to "radicalize" already existing enactivist or enactivist friendly approaches. Especially in the last section we explained how PPC accounts for what minds do. Later we have argued that this approach just as enactivist views - can be spelled out in both representationalist and REC terms. Consequently, we claim that Hutto's idea does not concern PPC (or enactivism) itself, it only concerns the way it is spelled out. For us there is no problem if PPC maintains its representationalist repertoire (with which it does so well). Therefore, we think the only option radical Enactivists have is to argue that REC's conceptual revolution is based essentially on methodological considerations. To maintain this view, RECers have to claim that REC is a methodological program applied to Enactivist approaches of basic minds.

Now, we have already argued that the consideration that primarily impacts cognitive science is clearly the enactivist idea that minds do not simply process information. Rather, organisms actively interact with their environment. Many proponents already assume that this is a non-intellectualist program (Varela, Thompson \& Rosch, 1991; Clark, 2016). That is to say that enactivism changes essentially how we evaluate the role of information. But is this enough for a revolution? Hutto explicitly denies this. For him, to be revolutionary - and implement a new methodological program - means that one also has to get rid of content and hence representations. This means, REC has to maintain that it is the way to spell out enactivism in the context of basic minds. Consequently, enactivist approaches are only capable of doing their job in a new manner when they are radicalized, i.e. when they do not refer to content/representations as essential.

Now, it is interesting to point out that what Hutto is trying to do in the context of cognition - namely to get rid of contents/representations and their semantic properties - is already discussed in the context 
of computation. Both, Piccinini (2006, 2015) and Fresco (2009), argue that the so called semantic view of computation ${ }^{19}$ should be replaced by a functional view that employs mechanistic explanations (Miłkowski 2016a). According to Piccinini a mechanistic explanation means, "[...] a description according to which a mechanism (e.g., the human body) has certain components (e.g., the heart), the components have certain functions (e.g., pumping blood) and are organized together (e.g., the heart is connected to the arteries in such and such a way), and the mechanism exhibits its capacities (e.g., blood circulation) because it is constituted by the relevant components, their functions, and their organization." (Piccinini, 2006, p. 206) The essential difference between both ideas is how they individuate computational states. As far as we can see, the mechanistic view gets rid of the essential role problematic semantic properties have and changes our view on how a specific computational state is picked out.

Now, it is interesting that this view is compatible with both representationalism and anti-representationalism. The essential claim is that even if there are semantic properties, state individuation is due to the underlying mechanism and not whether it is spelled out in representational or anti-representational terms. The same goes for enactivism ${ }^{20}$. What is new is the view that cognition is essentially described by a different mechanism, namely the organism's interaction with its environment. This is consistent with both representationalism and anti-representationalism. A good indicator may be that even Hutto thinks that a lot of his view is already used in cognitive science. He states that his content-less account of "[t]eleosemiotics borrows what is best from teleosemantics and covariance accounts of information [...]" (Hutto, 2011, p. 22). This means that the most important elements of his view have a striking resemblance with what is used in representationalism. In our view this is to be expected, since REC spells out the same underlying structure. As a consequence,

19 This view states that "[...] computation is best individuated by its semantic properties." (Fresco, 2009, p. 165).

20 We think that this is the actual methodological revolution. 
we think that REC cannot constitute a methodological revolution within enactivism and hence cognitive science ${ }^{21}$.

\section{Cognitive Science and the Long Revolution}

An alternative reconstruction of the recent history of Cognitive Science is that an anti-intellectualist research program was launched around the time of (Varela, Thompson \& Rosch, 1991) which continues to reverberate across the field. Its inheritors are multiple from work on the extended mind (Clark, 2008), to embodiment (Gallagher, 2005), from biology and life (Di Paolo, 2009) to methodology (Hutto \& Myin, 2013), to name just very few. At a certain point this radical character has deeply permeated cognitive science and informs much theorizing through its diverse fields. Hardly anyone now claims that the chief job of the brain is disinterested knowledge, all accept the central role of the brain in production of action within the organization and life of an agent. Claims for advancing theoretical purity 30 years on from the original statement of the revolution seems not to advance the cause very much. Indeed, it is questionable if the search for such theoretical unity does not in any case fly in the face of a more general heterogeneity in theory of cognitive science (Lakatos, 1970).

Alternatively, maybe it is up to PPC to finalize the revolution originally started by the enactivist program. This appears to be the sort of vision that informs Clark's interpretation (Clark, 2015b). At its heart, this program advances a new mechanistic interpretation of the brain which understands the brain as a distinctly biological entity ultimately constrained by the ongoing need to act and maintain homeostasis (Friston, 2009) which it does by predicting its future sensorimotor engagements. The irony is that this perspective seems to naturally incorporate a new way of thinking about representation which is highly congenial to at least

21 We think that, at this stage, even the supposably radical claims of "radical cognitive science" get watered down. Why should one think that anti-representationalist claims are automatically radical? In fact, anti-representationalism is a common view in Philosophy of Mind (see e.g. the consciousness debate (Block, 1996; Shoemaker, 1994)) prior to REC. Furthermore, Stich (1983) already in the eighties defended the view that cognitive psychology should subscribe to a syntactic theory of mind, claiming that semantic properties should not enter explanations in the context of the mental. 
a version of enactivism. If what we have argued is correct RECtification does not really add anything to this revolution in the current circumstances of cognitive science. It is just one way of spelling out a methodological program seeking to restrict the conceptual scope of theories which, to us, does not seem an obvious way of promoting conceptual experimentation. We prefer to let a hundred flowers bloom.

\section{Acknowledgements}

We would like to thank Michael Kirchhoff, Kenneth Aizawa, Simon McGregor, Max Jones, Marcin Miłkowski, Christopher Burr and Daniel Hutto for their valuable comments and discussion. We would especially like to thank Farid Zahnoun for his review (https: / www.academia. edu/s/64a2ee581c/review-enactivism-radical-enactivism-and-predictive-processing-what-is-radical-in-cognitive-science). Finally, we would also like to thank the Lisbon Mind and Cognition Group for their support.

Klaus Gärtner's work is endorsed by the CFCUL post-doctoral research fellowship (UID/FIL/00678/2013). Robert W. Clowes's work is endorsed by the FCT post-doctoral research fellowship (SFRH/BPD/70440/2010).

\section{References}

Bermúdez, J.L. (2003). Thinking Without Words. Oxford.

Block, N. (1996). Mental paint and mental latex. Philosophical Issues, 7, 19-49.

Brooks, R. (1995). Intelligence Without Reason. In L. Steels \& R. Brooks (Eds.), The Artificial Life Route to Artificial Intelligence: Building Embodied, Situated Agents. Hillsdale, New Jersey: Lawrence Erlbaum Associates.

Brooks, R., \& Stein, L.A. (1993). Building Brains for Bodies (1439). Retrieved from http://people.csail.mit.edu/brooks/papers/brains.pdf

Clark, A., \& Toribio, A.J. (1994). Doing without representing. Synthese, 10, 401-431.

Clark, A. (1997). Being There: Putting Brain, Body, and World Together Again. Cambridge, MA: The MIT Press. 
Clark, A. (2008). Supersizing the Mind: Oxford University Press.

Clark, A. (2012a). Dreaming the whole cat: Generative models, predictive processing, and the enactivist conception of perceptual experience. Mind 121 (483), 753-771. doi: 10.1093/mind/fzs 106.

Clark, A. (2012b). Whatever next? Predictive brains, situated agents, and the future of cognitive science. Brain and Behavioral Sciences 36(3), 181-253.

Clark, A. (2015a). Embodied prediction. In T. Metzinger \& J. M. Windt (Eds). Open MIND (7), 1-21. doi: 10.15502/9783958570115.

Clark, A. (2015b). Surfing Uncertainty: Prediction, Action, and the Embodied Mind: Oxford University Press.

Clark, A. (2016). Busting out: Predictive brains, embodied minds, and the puzzle of the evidentiary veil. Noûs 00:0, 1-27. doi:10.1111/ nous. 12140 .

Clowes, R.W. (2017). The Ipseity Disturbance Theory of Schizophrenia and Predictive Processing. In I. Hipólito, J. Gonçalves, \& J. G. Pereira (Eds.), Schizophrenia and Common Sense: explaining madness and social values. : Springer Mind Brain Studies.

Clowes, R.W., \& Chrisley, R. (2012). Virtualist Representation. International Journal of Machine Consciousness, 04(02), 503-522.

Clowes, R.W., \& Mendonça, D. (2016). Representation Redux: Is there still a useful role for representation to play in the context of embodied, dynamicist and situated theories of mind? New Ideas in Psychology, Special Issue on Representation(Special Issue: Explaining Representation), 26-47.

Degenaar, J., \& Myin, E. (2014). Representation-hunger reconsidered. Synthese, 191 (15), 3639-3648.

Di Paolo, E. (2009). Extended life. Topoi, 28(1), 9-21.

Di Paolo, E., \& Thompson, E. (2014). The enactive approach. The Routledge handbook of embodied cognition, 68-78.

Fletcher, P. \& Frith, C. (2009). Perceiving is believing: A Bayesian approach to explaining the positive symptoms of schizophrenia. Nature Reviews: Neuro-science 10, 48-58. 
Fodor, J. (1981). Representations, Cambridge: MIT Press.

Fresco, N. (2010). Explaining Computation Without Semantics: Keeping it Simple. Minds and Machines 20(2), 165-181. doi:10.1007/s11023010-9199-6.

Friston, K. (2009). The free-energy principle: a rough guide to the brain? Trends in Cognitive Sciences, 13(7), 293-301.

Gallagher, S. (2005). How the body shapes the mind. New York: Oxford University Press.

Gładziejewski, P. (2015). Predictive coding and representationalism. Synthese 193, 559-582 doi:10.1007/s11229-015-0762-9.

Haugeland, J. (1990). The intentionality all-stars. Philosophical perspectives, 383-427.

Hohwy, J. (2004). Top-down and bottom-up in delusion formation. Philosophy, Psychiatry, \& Psychology, 11 (1), 65-70.

Hohwy, J. (2013). The predictive mind: Oxford University Press.

Hohwy, J. (2014). The self-evidencing brain. Noûs. doi:10.1111/ nous.12062.

Hohwy, J., Roepstorff, A. \& Friston, K. (2008). Predictive coding explains binocular rivalry: An epistemological review. Cognition 108(3), 687-701.

Hume, D. (1978). A Treatise of Human Nature (Second Edition). Sir Lewis Amherst Selby-Bigge, first baronet \& P. H. Nidditch (eds.). Oxford University Press.

Hutto, D.D. (2011). Enactivism: Why be radical. Sehen und Handeln, 21-44.

Hutto, D.D. (2015). REC: Revolution Effected by Clarification. Topoi, 1-15. doi:10.1007/s11245-015-9358-8.

Hutto, D.D., Kirchhoff, M. D., \& Abrahamson, D. (2015). The enactive roots of STEM: rethinking educational design in mathematics. Educational Psychology Review, 27(3), 371-389.

Hutto, D.D., \& Myin, E. (2013). Radicalizing enactivism: Basic minds without content: MIT Press.

Hutto, D.D., \& Myin, E. (Forthcoming). Going Radical. Retrieved from https://www.academia.edu/15763822/Going_Radical?auto=download. 
Hutto, D.D., \& Satne, G. (2015). The natural origins of content. Philosophia, 43(3), 521-536.

Kriegel, U. (2002). PANIC Theory and the Prospects for a Representationalist Theory of Phenomenal Consciousness. Philosophical Psychology 15, 55-64.

Lakatos, I. (1970). Falsification and the Methodology of Scientific Research Programmes. In I. Lakatos \& A. Musgrave (Eds.), Criticism and the Growth of Knowledge, Cambridge University Press.

Margolis, E., \& Laurence, S. (1999). Concepts: core readings: Mit Press.

Miłkowski M. (2013). A mechanistic account of computational explanation in cognitive science. In M. Knauff, M. Pauen, N. Sebanz, I. Wachsmuth (Eds.), Proceedings of the 35th Annual Meeting of the Cognitive Science Society, Cooperative Minds: Social Interaction and Group Dynamics, Austin, TX: Cognitive Science Society, 3050-3055. Available online at: http://csjarchive.cogsci.rpi.edu/Proceedings/2013/papers/0545/paper0545.pdf.

Miłkowski, M. (2016a). Function and causal relevance of content. New Ideas in Psychology, 40, 94-102.

Miłkowski, M. (2016b). Unification Strategies in Cognitive Science. Studies in Logic, Grammar and Rhetoric, 48(61), 13-33.

O'Regan, J.K., \& Noë, A. (2001). A sensorimotor account of vision and visual consciousness. Behavioral and Brain Sciences, 24, 939-973.

Piccinini, G. (2006). Computation without Representation. Philosophical Studies 137(2), 205-241. doi:10.1007/s11098-005-5385-4.

Piccinini, G. (2015). Physical Computation: A Mechanistic Account. Oxford University Press.

Pitt, D. (2013). Mental Representation. In E. N. Zalta (ed.), The Stanford Encyclopedia of Philosophy Fall 2013 Edition, URL $=<$ http://plato.stanford.edu/archives/fall2013/entries/mental-representation/>.

Sacks, O. (2012). Hallucinations: Pan Macmillan.

Seth, A.K., Suzuki, K. \& Critchley, H.D. (2011). An interoceptive predictive coding model of conscious presence. Frontiers in Psychology 2, 395.

Shoemaker, S. (1994): Identity, Cause, and Mind. Cambridge University Press. 
Steels, L. (1997). Constructing and Sharing Perceptual Distinctions. Paper presented at the Proceedings of the European Conference on Machine Learning, Prague. Springer Verlag, Berlin.

Steels, L., \& Belpaeme, T. (2005). Coordinating Perceptually Grounded Categories through Language: A Case Study for Colour. Behavioral and Brain Sciences, 28(4), 469-489.

Stich, S. (1983). From Folk Psychology to Cognitive Science. The MIT Press.

Thagard, P. (1992). Conceptual revolutions. Princeton University Press.

Varela, F.J., Thompson, E., \& Rosch, E. (1991). The Embodied Mind. Cambridge, MA: MIT Press.

Watson, J.B. (1913). Psychology as the Behaviorist Views it. Psychological Review 20, 158-177. 